\title{
Violencia, migración y refugio: una mirada reflexiva a contribuciones sobre violencia estructural y movilidad geográfica
}

\author{
Eduardo Torre-Cantalapiedra* \\ Universidad Nacional Autónoma de México, México
}

\begin{abstract}
Resumen $^{* *}$
El objetivo de este trabajo es analizar las aportaciones que, desde el campo de los estudios de migración, se han realizado en torno a la noción de violencia estructural -introducida y popularizada por Johan Galtung-. Para su logro se examinan las investigaciones que manejan y/o reelaboran este concepto a fin de comprender los procesos migratorios en origen, tránsito y destino. Adicionalmente, se reflexiona en torno al caso de las caravanas migrantes centroamericanas en México a finales de 2018 y principios de 2019. Se concluye que el concepto de violencia estructural ha sido útil para analizar a profundidad los escenarios de violencia en los que están sometidos los migrantes y así poner a las legislaciones nacionales e internacionales en el punto de mira.
\end{abstract}

Palabras clave: Violencia estructural, migración internacional, refugiados, violencia postestructural, violencia legal, caravana de migrantes.

Violence, Migration and Refugee: A Reflexive Look at Contributions on Structural Violence and Geographic Mobility

\section{Abstract}

The objective of this paper is to analyze the contributions that, from the field of migration studies, have been made around the notion of structural violence-introduced and popularized by Johan Galtung. For its achievement, the investigations that handle and / or rework this concept are examined to understand the migratory processes in origin, transit and destination. Additionally, the case of the Central American migrant caravans in México at the end of 2018 and the beginning of 2019 is considered. It is concluded that the concept of structural violence has been useful to analyze deeply the scenarios of violence in which migrants are subjected and thus putting national and international legislations in the spotlight.

Key words: Structural violence, international migration, refugees, post-structural violence, legal violence, migrant caravan.

* UNAM. Programa de Becas Posdoctorales en la UNAM, Becario del Instituto de Investigaciones Jurídicas, asesorado por el doctor Enrique Mauricio Padrón Innamorato.

** Agradezco a Cynthia Rodríguez de Jesús por sus comentarios y sugerencias a una versión anterior de este trabajo. Agradecimiento que extiendo a los dictaminadores anónimos del mismo.

Recibido: 30 de enero de 2019

Aprobado: 13 de mayo de 2019 


\section{INTRODUCCIÓN}

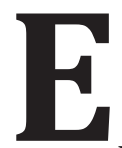

n los últimos años, numerosas investigaciones han analizado el binomio de violencia y migración en lugares de origen, ${ }^{1}$ tránsito, ${ }^{2}$ destino $^{3}$ y retorno ${ }^{4}$ en diversas geografías del mundo. El tema clave ha sido el fenómeno de las violencias que sufren los migrantes que ha sido examinado desde perspectivas cualitativas, cuantitativas y mixtas. Para el análisis de estas realidades se han desarrollado nuevas contribuciones teóricas y conceptuales que abonan al campo de los estudios sobre violencia.

Más allá del concepto de violencia directa o personal -la que comúnmente se conoce como tal一, los estudios migratorios han prestado especial atención a su acepción estructural, introducida y popularizada por el sociólogo noruego Johan Galtung. Asimismo, muchos de quienes emplearon el término violencia estructural no se limitaron a reproducir y retomar total o parcialmente el esquema de Galtung, sino que recuperaron las perspectivas de otros autores y realizaron sus propios desarrollos.

Varios de estos trabajos se centraron en el caso de las migraciones del denominado Triángulo Norte de Centroamérica (TNC) —conformado por Honduras, El Salvador y Guatemala. Algo que no resulta llamativo, dado que las poblaciones de estos países han padecido desde la década de los ochenta diversas formas de violencia- entre otras, conflictos bélicos y represión por parte de gobiernos totalitarios, la violencia de las pandillas y otros grupos delincuenciales que han quedado plasmados en algunos de las mayores ratios de violencia en el mundo-y los constantes abusos y agresiones que los migrantes sufren en tránsito por el territorio mexicano (Calva Sánchez et al.

1 Véanse, por ejemplo, Zolberg et al. 1989; y Massey, 2015; Torre Cantalapiedra y Giorguli, 2016; Huerta, 2017; Cordero Díaz y Garibo García, 2018; Cuellar Díaz y Moreno Hernández, 2018.

2 Véanse, por ejemplo, Nevins 2003, 2005; Alonso Meneses, 2005; Jácome, 2008; Spener, 2008; Slack y Whiteford, 2010; Vogt, 2013; Martínez et al. 2014; Willers, 2016, Yee Quintero, 2016, Cortés, 2018.

3 Véanse, por ejemplo, Abrego y Menjívar, 2011; Menjívar y Abrego, 2012; Izcara Palacios, 2012.

4 Véase, por ejemplo, Rocha Ramírez y Vera Martínez, 2016. 
2015; , 2017; REDODEM, 2017; París Pombo, 2017; Díaz y Moreno Hernández, 2018; El Colef, 2018).

El objetivo de este trabajo es analizar las aportaciones que se han realizado en torno a la noción de violencia estructural desde el campo de los estudios de migración. ${ }^{5}$ Para su logro el trabajo está divido en dos secciones: primero, se analiza el esquema de violencia personal y estructural propuesto por Galtung $(1995,2003) .{ }^{6}$ Se enfatiza la acepción estructural de violencia y se destaca la amplitud de la misma, esta característica puede considerarse, paradójicamente, su mayor ventaja y a la vez su mayor inconveniente.

Segundo, se examinan las contribuciones que manejan y/o reelaboran el concepto de violencia estructural para lograr una mejor comprensión de la violencia presente en los procesos migratorios en diferentes momentos de las trayectorias de los migrantes. Esto es, la violencia estructural: I) como motivo de la movilidad; II) que los migrantes irregulares sufren en países de tránsito y en el cruce de fronteras internacionales; III) como generadora de violencia postestructural; IV) y como componente de la violencia legal que dificulta la integración de los migrantes y sus familias en los países de destino. Adicionalmente, se reflexiona sobre el reciente caso de las caravanas de migrantes centroamericanas que transitaron por México a finales de 2018 y principios de 2019 - compuesta principalmente por migrantes del TNC que escaparon de la situación de violencia y pobreza que vivían en sus países de origensiguiendo la estela de los hallazgos de las investigaciones examinadas.

A modo de conclusión, se presentan algunas reflexiones en torno al concepto de violencia estructural en los estudios de migración que pueden servir de guía para futuras investigaciones. Aunque el concepto de violencia estructural es complejo de delimitar, ha sido de gran utilidad para analizar a profundidad la violencia a la que están sometidos los migrantes. En particular, el análisis de las políticas y legislaciones migratorias y sus efectos sobre la vida de los migrantes constituye un nicho para futuras investigaciones que observen la

5 Se realizó una exhaustiva revisión de la literatura académica sobre migración, con especial atención a los movimientos humanos dentro del sistema migratorio que de acuerdo con Giorguli et al. (2018) conforman los tres países de América del Norte (México, Estados Unidos y Canadá) y los tres países del TNC, incluyendo libros y artículos académicos -impresos y/o disponibles de manera electrónica一, que abordaron la cuestión de la violencia estructural.

6 Publicados originalmente en los años 1969 y 1990, respectivamente. 
realidad a través de la lente que proporciona la violencia estructural y bajo el paraguas del paradigma de la teoría crítica.

\section{VIOLENCIA ESTRUCTURAL}

Según (Galtung, 1995: 314), “la violencia está presente cuando los seres humanos se ven influenciados de tal manera que sus realizaciones efectivas, somáticas y mentales, están por debajo de sus realizaciones potenciales". De acuerdo con el esquema que despliega el autor, esta concepción amplia ${ }^{7}$ de violencia engloba: la violencia personal -0 directa - y la violencia estructural -o indirecta. ${ }^{8}$ Galtung denomina violencia estructural a aquella que, en contraste con la violencia personal- en la cual hay un actor reconocible, no está cometida, o al menos no necesariamente, por un actor o actores identificables.

Puede no haber en la estructura ninguna persona que dañe directamente a otra persona. La violencia está edificada en la estructura, y se manifiesta como un poder desigual, y consiguientemente, como oportunidades de vida distintas (Galtung, 1995: 320).

Este carácter indirecto de la violencia estructural supone que los investigadores deben afrontar la ardua tarea de demostrar que los resultados observados corresponden a la existencia de tal tipo de violencia.

Podemos considerar que no es tan relevante evidenciar a los perpetradores puesto que siempre se puede llegar a la conclusión de que quienes generan y reproducen materialmente las estructuras más concretas donde los autores concretos son discernibles, lo hacen en función de estructuras represivas más amplias en las cuales todos - como sociedad - seríamos responsables. Resulta más importante dar cuenta de la manera en que se generan y reproducen las

7 Galtung (1995) considera fundamental disponer de una concepción amplia de violencia en consonancia con el hecho de que define la paz como la negación de violencia; puesto que, el concepto de paz no debe acotarse a la ausencia de violencia directa.

8 Galtung (2003) incorpora una tercera categoría global de violencia, la cultural, que junto a la directa y la estructural son colocadas por el autor en los ángulos del denominado "triángulo de la violencia". Según el autor, las tres categorías se relacionan entre sí, de tal modo, que hay relaciones de causalidad en seis direcciones y los ciclos que las interconectan pueden comenzar en cualquiera de los puntos. "En el fondo está el constante caudal de la violencia cultural a lo largo del tiempo, de cuyo sustrato las otras dos formas pueden extraer nutrientes" (Galtung, 2003: 13). En este sentido, "la cultura sermonea, enseña, amonesta, incita y nos embota para que aceptemos la explotación y/o represión como algo normal y natural, para que no las veamos en absoluto (en especial la explotación)" (Galtung, 2003: 13). 
estructuras represivas para cambiarlas y con ello, evitar sus nefastas consecuencias.

En el esquema Galtung, la violencia estructural se caracteriza por: I) Tener una vinculación indirecta con el objeto. En el caso de la violencia personal el vínculo entre el sujeto y el objeto (siendo personas tanto el sujeto como el objeto) se establece de manera directa, mientras que en el caso de la violencia estructural se establece un vínculo indirecto y estructural. La violencia golpea a los seres humanos "indirectamente a causa de la existencia de estructuras represivas (...) sostenidas por la acción acumulada y concertada de los seres humanos" (Galtung, 1995: 336). II) Estar más enfocada en las consecuencias objetivas que a determinar la responsabilidad de ciertos actores - en el caso de que fuera posible. III) Estrechamente relacionada con el elemento anterior, se refiere a una acción que no es necesariamente deliberada. La distinción entre violencia deliberada y no deliberada es relevante cuando se quiere dilucidar una culpa, pero no cuando se pretende enfatizar las consecuencias. IV) Ser evitable. V) Ser relativamente estática- varía menos a lo largo del tiempo histórico que la violencia directa. VI) Estar naturalizada y pasar desapercibida. g) Relacionarse de múltiples maneras con la violencia directa. VII) Ser reproducible a través de diversos mecanismos.

Paradójicamente, el concepto de violencia estructural de Galtung tiene como principal problema su mayor virtud: la amplitud. La noción de violencia estructural, como parte más difusa del concepto amplio de violencia, podría usarse - y se usa - para referir infinidad de situaciones como violencia. Esto implica una extensión semántica del concepto tradicional de violencia, sin que existan reglas precisas que indiquen en qué casos resulta apropiado el uso del término y en cuáles no. ${ }^{9}$ Esta flexibilidad puede saturar el contenido del propio concepto, por tanto, inutilizándolo como herramienta analítica.

Resulta oportuno traer a colación la observación crítica que hace Cody (citado en La Parra y Tortosa, 2003) respecto este concepto de violencia estructural, al señalar que su mayor problema es la sugestión de que diversos problemas sociales, aparentemente diferentes entre sí, son la misma cosa y tendrían que ser abordados de igual manera. En este trabajo se denomina "problema de amplitud" al conjunto

9 Por ejemplo, en un determinado caso se puede señalar como una situación de violencia estructural desde las muertes de migrantes que enfrentan políticas migratorias restrictivas hasta imposibilidad de que los migrantes se integren plenamente en la sociedad receptora debido a ciertas legislaciones en su contra. 
de problemáticas conceptuales descritas respecto a la acepción estructural de violencia. Por lo tanto, resulta oportuno, al menos en el ámbito académico, justificar la atribución/extensión que se haga de las connotaciones y carga valorativa del concepto violencia en su acepción común - violencia directa o personal- a otras situaciones distintas, como las sean consideradas de violencia estructural. Al respecto, conviene destacar la advertencia del propio Galtung (1995: 314) en cuanto a la concepción amplia de violencia manejada: "debería ser una extensión lógica, no simplemente una lista de cosas indeseables."

En cualquier caso, lejos de considerar estéril el concepto de violencia estructural, algunos autores no dudan en afirmar su utilidad. La Parra y Tortosa (2003: 61-62) dan cuatro argumentos sobre la conveniencia del uso del término violencia estructural puesto en relación con la violencia directa - frente al uso de otros - como el de desigualdad social: I). Ambos términos, violencia estructural y directa, constituyen motivos por los que muchas personas no pueden satisfacer sus necesidades humanas más elementales. II). Permite, analizar las interrelaciones entre violencia estructural y violencia directa. III). Incorporar los mecanismos de ejercicio del poder que privan a las personas de poder cumplir sus necesidades humanas básicas. IV). Contiene una carga valorativa y explicativa determinante: la deprivación se define como el resultado de un conflicto entre dos o más partes en el que el reparto, acceso o posibilidad de uso de los recursos es resuelto sistemáticamente a favor de alguna de las partes y en perjuicio de las demás. Al calificar esta situación como violenta se descarta la posibilidad de buscar mecanismos de legitimización de la desigualdad en la insatisfacción de las necesidades.

Este último aspecto evidencia el calado político e ideológico del cambio semántico antes mencionado. En consecuencia, el hecho de afirmar que existe violencia en una determinada situación, en vez de desigualdad u otro término similar, produce de modo automático una repulsa mucho mayor ante un mismo hecho. En este sentido se puede considerar que el uso más legítimo del término es el que se hace en los análisis sobre la opresión de los más débiles y desfavorecidos en una sociedad.

La noción de violencia estructural en Galtung ha resultado de interés para los investigadores quienes lo han manejado, interpretado y reelaborado de muy diversas maneras — que incluso llegan a ser contradictorias entre sí y a diferir del esquema propuesto por el 
sociólogo noruego. Así, en lo que respecta a su delimitación, algunos autores lo acotan con precisión mientras que otros mantienen la ambigüedad del esquema de Galtung, por lo tanto, persistiendo el problema de amplitud-. Asimismo, existen importantes divergencias en la manera en que las diversas investigaciones: consideran relevante o no la búsqueda de los perpetradores de la violencia estructural, entienden de una manera u otra cómo se producen las relaciones entre violencia directa y violencia estructural, toman en cuenta diferentes las estructuras/mecanismos de la violencia estructural que tienen en cuenta, entre otros.

\section{LA VIOLENCIA ESTRUCTURAL EN LOS ESTUDIOS MIGRATORIOS}

La violencia "acompaña" de manera continua a miles de migrantes a lo largo de su experiencia migratoria (Nájera Aguirre, 2016; Willers, 2016; París Pombo, 2017; Cordero Díaz y Garibo García, 2018). No obstante, lo habitual es que las investigaciones analicen un sólo momento y espacio de la trayectoria migratoria. Tomando en consideración este hecho, y por claridad en la exposición, en este apartado se analiza la violencia estructural: primero, como motivación de la migración; segundo, en el tránsito de territorios y en el cruce de fronteras internacionales; tercero, en relación con la violencia postestructural que padecen en el tránsito; y cuarto, en relación con la violencia legal que dificulta la integración en la sociedad de acogida.

\section{Motivación de la migración}

En un exhaustivo trabajo sobre la violencia y los refugiados en los países en vías de desarrollo, Zolberg, Suhrke y Aguayo (1989) retoman el concepto de violencia estructural ${ }^{10}$ en el sentido de una intensa y sistemática opresión económica presente en muchos países -por tanto, de una forma mucho más acotada y específica que Galtung (1995). Los autores consideran que quienes padecen violencia estructural están fuera de los flujos migratorios internacionales - particularmente ausentes en las corrientes de solicitantes de asilo-, en tanto que, por lo general, carecen de los recursos económicos necesarios para moverse; y que, de producirse la movilidad, esta tomará principalmente la forma

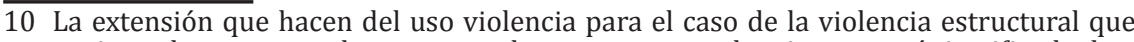
oprime a las personas de extrema pobreza o cercana a la misma estaría justificado desde el momento en que las consecuencias de esta se asemejan a las de la violencia directa. 
de migración interna dentro del país puesto que requiere de menos recursos.

Zolberg et al. (1989) señalan que la regla para tratar a quienes padecen violencia estructural - esto es, violencia económica- es, en primer lugar, que sean ayudados en sus países de origen; para evitar que países vecinos - muchas veces en vías de desarrollo- se vean sobrecargados por afluencia de personas en situaciones necesitadas y no promover la reestructuración internacional de poblaciones -algo que señalan es políticamente inaceptable en un mundo divido en estados-nación-. Postura que concuerda con la defensa de Pogge (2010) de centrar los esfuerzos en mejorar las condiciones de vida locales de los pobres globales mediante reformas institucionales internacionales, en vez de dedicarlos a promover la admisión de más extranjeros en nuestras sociedades ricas.

Hay que tener en cuenta el hecho de que Zolberg et al. (1989) retoman la noción de violencia estructural limitándola a la dimensión económica y debatiendo en concreto las situaciones de extrema pobreza. Dejando de lado las dimensiones no económicas, si considerásemos incluir en un sentido más general la migración de los pobres, excluidos y desempleados - no necesariamente en pobreza extrema- dentro de quienes sufren la violencia estructural, entonces este sería considerado uno de los más importantes motivos por los que las personas migran en el mundo.

En este sentido, es oportuno traer a colación el señalamiento de Delgado Wise et al. (2013) respecto a que la globalización neoliberal ha implicado cambios estructurales que desarticulan y desmantelan los sistemas de producción, financieros, comerciales y de servicios, lo que compele a grandes volúmenes de personas — que según Wise y sus colegas deben ser incluidas en el fenómeno de las migraciones forzadas-, a emigrar en busca de mejor calidad de vida para ellos y sus familias. De manera muy similar, Slack y Whiteford (2010) argumentan que las políticas económicas han provocado que sean abolidas las formas de la agricultura de subsistencia en México y en países de Centroamérica, lo que ha desencadenado migraciones internas e internacionales; por lo que explícitamente consideran que es la violencia estructural el motivo de tales migraciones.

Zolberg et al. (1989) discuten sobre la no inclusión de las personas que sufren violencia estructural bajo el paraguas protector que constituye la categoría legal de refugiado. En este sentido, evidencian 
que la situación de extrema pobreza provoca situaciones de riesgo para la vida del mismo calibre que situaciones de violencia física que sí están amparadas bajo la consideración de refugiado. Entonces podemos preguntarnos, en el mismo sentido que hace Espinar Ruiz (2010), ¿Por qué no ampliar la categoría legal de refugiado precisamente a través de la noción de violencia estructural? En la realidad, la ampliación de la categoría legal de refugiado parece poco factible, por el contrario, las tendencias recientes son a la restricción y la exclusión de quienes necesitan protección internacional y a la falta de solidaridad con los mismos (Crisp, 2003; Castles y Miller, 2004; Arango, 2016).

Zolberg et al. (1989) presentan de manera crítica las estructuras económicas que de alguna manera generan la represión de quienes padecen la violencia estructural y su exclusión del sistema de refugio, llámese la funcionalidad de los más pobres en el sistema económico capitalista. Sin embargo, el análisis que hacen del sistema político internacional se muestra menos crítico. No por ello dejan de evidenciar limitaciones políticas a la migración internacional al referir a la organización del mundo en estados-nación, lo que para algunos autores es una cuestión central. ${ }^{11}$

Las migraciones centroamericanas hacia Estados Unidos, que se producen en grandes volúmenes, particularmente desde los años noventa, ponen de manifiesto que en la actualidad, tanto la violencia directa como la violencia estructural —entendida como pobreza que no permite cubrir las necesidades básicas desde un punto de vista económico- son ambas motivaciones de la migración y que las decisiones de migrar de muchos migrantes se producen por la confluencia de ambas violencias — que además están interrelacionadas entre sí-y de otros factores (por ejemplo, redes sociales).

En casos como el de las recientes migraciones procedentes de TNC, la inclusión de la violencia estructural —en el sentido acotado a la pobreza extrema- a la categoría legal de refugiado supondría que muchos de quienes migran tendrían la oportunidad de obtener el estatus legal de refugiado. Así, por ejemplo, de acuerdo con cifras del Instituto Nacional de Estadística de Honduras el 42.5 por ciento de las personas viven en hogares en pobreza extrema (INE, 2018). Miles de personas en esta situación lograron llegar a Estados Unidos empleando diversas estrategias migratorias en tránsito -como

11 Entre ellos Spener (2008), cuyo desarrollo teórico-conceptual será analizado en el siguiente apartado. 
recorrer gran parte del camino a pie, apoyarse en la red de albergues y refugios situados a lo largo del territorio mexicano o participar en caravanas de migrantes como las de finales de 2018 y comienzos de 2019-..$^{12}$

A pesar de los esfuerzos de los varios cientos de participantes en las caravanas migrantes de Centroamérica, quienes de ellos solicitaron asilo en las garitas fronterizas o una vez en territorio estadounidense, enfrentan un sistema de refugio con altísimas tasas de rechazo para los solicitantes de los países del TNC (TRAC, 2018). Además, las sucesivas administraciones estadounidenses han impuesto un sistema de turnos por el cual quienes desean hacer una solicitud de asilo deben esperar semanas en ciudades fronterizas donde su integridad física está en riesgo e incluso sus vidas (Heyman y Slack, 2018). Con el añadido de que de acuerdo con la nueva política de la administración del presidente Donald Trump, muchos de los solicitantes hondureños posiblemente serán devueltos al territorio para esperar durante meses sus audiencias para el asilo en Estados Unidos, lo que algunos analistas consideran un equivalente de facto de México como tercer país seguro (Castañeda, 2019, 27 de enero).

En México, además de aceptar esta decisión "unilateral" de las autoridades estadounidenses, la nueva administración (2018-2024), del presidente Andrés Manuel López Obrador, ha considerado que la mejor manera de abordar el fenómeno de la migración centroamericana que huye de la pobreza y la violencia es el apoyo del desarrollo económico y social de los países de origen y dar un trato humanitario a los migrantes que llegan hasta sus fronteras. A lo largo de los próximos años se podrá observar la manera en que estos ejes se hacen o no efectivos para atender el fenómeno de la migración centroamericana en sus países de origen y en México.

\section{Tránsito de territorios y cruce de fronteras}

Jácome (2008) analiza que la violencia que los migrantes - particularmente, los centroamericanos- experimentan al transitar de manera clandestina por México en su camino hacia Estados Unidos, concluye que la misma debe ser entendida como un caso de violencia estructural; pues éste permite observar cómo las estructuras de violencia se construyen en detrimento de un grupo social de

12 Véanse, por ejemplo, Silva Hernández, 2015; Yee Quintero y Torre Cantalapiedra, 2016; París Pombo, 2017; Torre Cantalapiedra y Mariscal Nava, (s.f.) 
transmigrantes y una mayor comprensión de la violencia que sufren los migrantes en su camino a Estados Unidos.

Jácome retoma los conceptos de violencia personal y violencia estructural de Galtung, destacando el carácter directo e indirecto de cada uno de ellos respectivamente. Asimismo, complementa su marco analítico con los desarrollos teóricos de violencia de Farmer (2005), quien según Jácome (2008), a diferencia de Galtung, pone especial énfasis en los agentes que crean las estructuras socioeconómicas. Aunque el autor, está en lo cierto al señalar que el esquema de Galtung presta menos atención a los agentes perpetradores que a las consecuencias objetivas, considero que esto no significa que las elaboraciones realizadas en torno a su esquema de violencia - no pueda desarrollarse prestando mayor atención a los mismos- como veremos a continuación en los trabajos: Nevins (2005) y Spener (2008).

Las manifestaciones de violencia estructural respecto a la migración en tránsito por México para Jácome (2008) son la marginalización, la pobreza, el hambre y los riesgos para la salud. De acuerdo con este autor, la marginalización se refiere al hecho de que la persecución de las autoridades a los migrantes y las extorsiones que realizan a los mismos disminuyen la capacidad de agencia de los migrantes y los conduce a las rutas y medios de transporte más peligrosos. Asimismo, considera que la restricción de la agencia y la marginación a su vez coadyuva a que se generen otras formas de violencia indirecta contra los migrantes: pobreza, el hambre y los riesgos para la salud.

Jácome (2008) considera que la violencia directa e indirecta están entrelazadas, y que la una tiene implicaciones sobre la otra de manera recíproca; así, en muchos casos, la violencia estructural hace posible que la violencia directa pueda ser generadora de violencia indirecta; su entrelazamiento, hace que las muestras de violencia directa queden impunes y sean sistemáticas. Poner en relación la violencia directa con la estructural permite dar cuenta que las diferentes formas de violencia no se producen de forma aislada las unas de las otras.

Además, analiza las estructuras subyacentes que perpetúan la violencia; encuentra ocho fuerzas sociales, económicas y políticas que estructuran y permiten que la violencia tenga lugar, las cuales 
son complementarias y están interrelacionadas. ${ }^{13}$ Una de ellas la constituyen las prácticas migratorias de México.

Si bien, en primera instancia el desarrollo teórico-conceptual de Jácome (2008) no resuelve la problemática de la amplitud, en segunda instancia, el análisis acota las manifestaciones de violencia estructural a cuatro conceptos entre los que destaca la marginalización. En cualquier caso, por más sugerente que resulte la propuesta, caben realizar algunos cuestionamientos básicos ¿Por qué esos cuatro conceptos y no otros? ¿De qué manera se llega a la marginalización como concepto clave?

Son varios los trabajos que analizan la violencia que sufren los migrantes en su intento de cruzar la frontera entre México y Estados Unidos. Algunos de ellos han retomado el esquema de violencia de Galtung, primando la noción de violencia estructural, para analizar la violencia a la que están sometidos los migrantes, sus orígenes y consecuencias.

Nevins $(2003,2005)$ argumenta que las muertes de migrantes acaecidas en la frontera son generadas por violencia estructural; suponiendo que uno es responsable de las consecuencias predecibles de sus propias acciones, el autor concluye que los actores estatales son quienes ejercen esta violencia amparados por las leyes, construyen los límites e ilegalizan a los inmigrantes de tal modo que hacen que tales muertes sean inevitables. Nevins considera que, si bien la muerte de un migrante en particular o el número exacto de muertes no es algo que se pueda predecir, por el contrario, sí lo es que se produzca un importante número de muertes entre la población migrante; y que, por tanto, son muertes destinadas a suceder como resultado de estructuras y acciones que no se perciben como tales.

Como se puede apreciar Nevins hace un uso del concepto de violencia estructural en un sentido muy acotado, puesto que alude específicamente a las muertes que se producen en los intentos de cruce - ya sea que estas ocurran "mediadas" por la violencia personal o no-, lo que justifica emplear el termino violencia (estructural).

13 I) Las prácticas migratorias de México; II) las actitudes históricas respecto a la migración; III) xenofobia en contra de los migrantes; IV) la influencia de Estados Unidos en la política migratoria; V) el sistema judicial mexicano; VI) el incremento de la presencia de tráfico de drogas; VII) pobreza, privación de derechos, en la población local a lo largo de la ruta y VIII) las condiciones socioeconómicas en los países emisores" (Jácome, 2008: 26, traducción propia). 
Asimismo, su argumentación se apoya en la Declaración Universal de Derechos Humanos, que incluye el derecho a la vida, a la libertad, etcétera.

Por lo tanto, la negación de estos derechos, o más específicamente, la negación de los medios para realizar estos derechos (en este caso, la libertad de movimiento) parecen ajustarse a la noción de violencia de Galtung como aquella que impide alcanzar metas sociales realizables que la mayoría considera deseables (Nevins, 2005: 17).

Frente al esquema de Galtung que se resta importancia a la determinación de los perpetradores de la violencia, Nevins evidencia a quienes considera principales responsables de las muertes en la frontera: los actores estatales; mostrando el mecanismo/instrumento por el cual se genera/perpetúa la violencia estructural: la ley. La opción de hacer explícito los actores y mecanismos permitiría realizar acciones para revertirlas las situaciones de violencia, pero también supone que se deben establecer relaciones de causalidad defendibles desde un punto de vista académico. En este caso, la argumentación del autor parece insuficiente para poder establecer tal autoría con taxatividad. Asimismo, Nevins $(2003,2005)$ utiliza argumentos, que podrían llegar a considerarse falaces por hiperbólicos, por ejemplo, aquellos que hablan de predestinación e inevitabilidad.

Aunque el problema de amplitud no queda explícitamente resuelto, la noción de violencia estructural es traído a colación para comprender la muerte de los migrantes cuando cruzan de manera clandestina la franja fronteriza, esto es, tiene un uso específico para referir a la más grave consecuencia para la vida humana, lo que hace plenamente justificable la extensión del término violencia a estos casos.

Reineke et al. (2014) emplean el esquema de violencia de Galtung para señalar que la violencia que enfrentan los centroamericanos en México y Estados Unidos se puede resumir como violencia estructural en el cruce a Estados Unidos y violencia directa en México. Estos autores señalan que el concepto de violencia estructural acuñado por Galtung refiere a procesos sociales, legales o de otra forma estructurados que conducen a niveles desproporcionados de riesgos para algunos, mientras que "protege" a otros. Una interpretación ad hoc para su análisis de violencia en tránsito y cruzando fronteras que no es lo suficientemente acotada para resolver el problema de amplitud. 
Al igual que Nevins (2003; 2005), Reineke y Martínez (2014) consideran que las muertes de migrantes debido a la exposición a los elementos de la naturaleza en el cruce de la frontera México-Estados Unidos corresponden a la existencia de violencia estructural, pero, a diferencia de aquel, no buscan quienes son los perpetradores de esta clase de violencia.

Por otra parte, Reineke y Martínez (2014) no sólo reflexionan sobre la violencia en el cruce de la frontera México-Estados Unidos, sino que analizan el cruce de la frontera Guatemala-México y el tránsito por México. Consideran que la violencia que sufren los migrantes - destacando particularmente las muertes - corresponde mayoritariamente a la violencia directa: los homicidios. Aunque no descartan la violencia estructural, pues existen muertes debido a la exposición a diversos riesgos como los ahogamientos en el Río Suchiate, las caídas accidentales de los trenes, entre otras.

Ya sea quede reflejado de manera implícita, se puede observar que la violencia directa y la violencia estructural operarían cada una por separado. Esta manera de entender el esquema de Galtung puede resultar demasiado simplista para el caso de la migración centroamericana en tránsito y cruzando fronteras, ya que como lo señalan Jácome (2007) y Spener (2008) las situaciones de violencia estructural y directa están entrelazadas.

Spener (2008) analiza los discursos dominantes sobre la migración clandestina de mexicanos hacia Estados Unidos apoyada por coyotes, dando cuenta de las inconsistencias de estos y proponiendo discursos alternativos. Asimismo, en su trabajo señala que los actos de violencia personal y directa en contra los migrantes realizados por los coyotes pueden ser mejor entendidos en el contexto de la violencia estructural generada por los estados como parte de un sistema de apartheid global y se muestra que actores están produciendo/reproduciendo las estructuras que generan la violencia estructural.

Spener (2008: 130) señala que el aparheid global:

Enfatiza de qué manera la mala distribución de los recursos y del bienestar en el mundo está fuertemente correlacionado con la raza y la nacionalidad" y que éste "desempeña un papel crucial en el mantenimiento de las inequidades globales en la medida en que mantiene espacios sociales, políticos y económicos separados en el sistema mundial y restringe además la capacidad de los residentes empobrecidos, de trasladarse de una región a otra en busca de ingresos más altos y mejores estándares de vida. 
Según Spener (2008: 143):

El aparato de control de la inmigración y de la frontera que posee el departamento de seguridad nacional, así como los fiscales y cortes estadounidenses que procesan a quienes, entre los migrantes practican la resistencia de la hormiga (esto es, llevan a cabo estrategias clandestinas autónomas de cruce de fronteras), constituyen elementos indispensables en la institucionalización del apartheid global, es decir, forman parte de la violencia estructural que se impone a los migrantes.

La propuesta de este autor se podría esquematizar en tres planos entrelazados: I) político-legal (la forma en que está organizado política y legalmente el mundo: el apartheid global), II) discursivo (los discursos que reproducen y mantienen el apartheid global) y III) operativoinstitucional (como parte institucionalizada en un determinado estado nación del apartheid global). Todos dificultan que los migrantes logren desarrollar todo su potencial y cubrir sus necesidades básicas acudiendo a la migración internacional y ejerciendo la estrategia de la hormiga.

En lo que respecta a la relación entre violencia estructural y violencia directa, Spener (2008) señala que la violencia estructural es un contexto que favorece a la violencia directa que los coyotes ejercen sobre los migrantes. Así la intensidad de la vigilancia fronteriza afecta las relaciones entre los migrantes y coyotes. Por ejemplo, dado que los encuentros con las autoridades son cada vez más perjudiciales para los coyotes, favorece que estos últimos tengan un comportamiento más violento sobre los migrantes, en pro de evitar tales encuentros o minimizar que estos les afecten negativamente

Las propuestas de Jácome (2008), Nevins (2003, 2005), Spener (2008) y Reineke y Martínez (2014) permiten entender mejor la violencia a la que se enfrentan los migrantes procedentes del TNC en tránsito por México, que no sólo es el resultado del encuentro con criminales y agentes corruptos, sino que responde a un escenario de violencia estructural que: I) implica marginalización, empobrecimiento, debilitamiento, etcétera, de los migrantes, II) que facilita a su vez el ejercicio de la violencia personal sobre los migrantes y III) también causa muertes, enfermedades, daños físicos y sicológicos a los migrantes en tránsito por el territorio y cruzando las fronteras sin que sea perpetrado directamente por persona alguna. De diferentes maneras, todos estos autores refieren a que las prácticas de 
las autoridades migratorias y agentes del estado alimentan la violencia estructural.

Lo anterior permite dar cuenta de que el reciente fenómeno de las caravanas de migrantes centroamericanos no sólo responde a las condiciones de vida en sus países de origen, sino que permite que los migrantes hagan frente de mejor manera a la violencia que experimentan en México, tanto personal como estructural. Durante décadas, las autoridades mexicanas no han respondido de manera adecuada a la situación de violencia que viven los migrantes, primaron la política de control -establecieron una frontera vertical- sobre la protección de los migrantes (Torre Cantalapiedra y Yee Quintero, 2018).

En sus primerísimos compases de gobierno la administración de López Obrador (2018-2024) ha tenido luces y sombras en cuanto a sus actuaciones respecto a los participantes en tales caravanas. Por un lado, la aceptación de que los solicitantes de asilo sean devueltos a México mientras son resueltos sus casos, amerita ser analizado desde el punto de vista de la violencia estructural más que como una medida humanitaria. Por otro, las iniciativas de otorgar a los migrantes documentos de diversas clases, por ejemplo, visas humanitarias, que les permiten circular libremente sin ser perseguidos por las autoridades migratorias, son importantes para aminorar la violencia estructural y directa que los migrantes procedentes del TNC padecen a su paso por México.

\section{Violencia postestructural}

En los contextos del cruce de la frontera entre México y Estados Unidos y de tránsito por el territorio mexicano se ha empleado un concepto de violencia que es en parte producto de la situación de violencia estructural que experimentan los migrantes en tales circunstancias. Slack y Whiteford (2010) acuñan el término violencia postestructural para describir las reacciones de las personas ante la violencia estructural, acciones que pretenden conducir a aminorar la situación de vulnerabilidad y marginalidad en la que se encuentran. De acuerdo con estos autores la violencia postestructural no significa que las condiciones de violencia estructural terminen, de hecho, las diferentes formas de violencia se producen de manera simultánea produciendo el escenario de violencia. 
Slack y Whiteford (2010) despliegan este concepto para dar cuenta de las circunstancias en que se ven envueltos los migrantes en condiciones de vulnerabilidad, sin protección económica y social, en la región fronteriza, donde inevitablemente se involucran con guías, asaltantes y traficantes de drogas. Las decisiones que toman las personas - aunque en buena medida están restringidas - los lleva a involucrarse en los sistemas de violencia.

En un estudio en que se entrevistó a migrantes centroamericanos secuestrados y forzados a participar en actividades ilícitas en México, Izcara Palacios (2016) retoma el concepto de violencia postestructural de Slack y Whiteford y lo (re)define señalando que aquellas situaciones en las que la víctima se convierte en verdugo como única forma de sobrevivir en un entorno de violencia, donde las acciones del individuo están regidas por su lucha de salir con vida de la situación. Añade, que este tipo de violencia quita a la víctima de su inocencia, lo carga de culpabilidad y tiene como precondición la violencia directa extrema; según este autor un migrante pacífico no se convierte en un criminal sanguinario sin que medie una violencia que amenace su vida. La violencia es, por tanto, una forma en la que los grupos delictivos reclutan a los migrantes capturados mediante la transformación de sus víctimas en verdugos forzados, a su vez, a emplear la mayor cantidad de violencia posible para sobrevivir.

Ambas conceptualizaciones de violencia postestructural se diferencian primordialmente en que mientras Slack y Whiteford (2010) consideran que la violencia estructural, en cuanto a la marginalización y vulnerabilidad que conllevan, es quien genera violencia postestructural y dejan más abiertas las posibilidades de acción de los migrantes — no sin un alto grado de ambigüedad-, Izcara Palacios (2016) destaca a la violencia directa en grado extremo como precondición de la violencia postestructural, descrita esta última en términos mucho más concretos: la conversión de víctima en verdugo. Las dos dan una explicación de cómo los migrantes se involucran con criminales y como ellos mismos son quienes acaban por ser generadores de violencia.

Retomando en particular la propuesta de Slack y Whiteford (2010), podemos entender que la violencia postestructural como un mecanismo a través del cual quienes sufren la violencia estructural pueden resultar en productores de violencia directa. Por tanto, el esquema de estos autores problematiza el esquema de violencia de 
Galtung (1995) en el cual se entiende que la violencia estructural produce exclusivamente víctimas.

\section{Violencia legal e integración}

Menjívar y Abrego (2012) analizan los efectos dañinos que las leyes pueden tener en dificultar las trayectorias de incorporación de los migrantes en Estados Unidos, con especial atención al caso de la población centroamericana. Las autoras acuñan el término "violencia legal" para referirse a estos efectos que las legislaciones incorporaran en sus textos; los cuales al mismo tiempo que pretenden proteger derechos y procurar el bien general, incrementan las prácticas que dañan a un grupo social en particular y posibilitan varias formas de violencia en contra del grupo al que van dirigidas. Las autoras justifican el uso de este término en el hecho de que la violencia en este caso está integrada en las prácticas legales, sancionada, activamente implementada a través de procedimientos formales y legitimada $-\mathrm{y}$ consecuentemente - vista como algo "normal" y natural porque "es la ley" (Menjívar y Abrego, 2012: 1386, traducción propia).

Menjívar y Abrego (2012) señalan que el concepto de violencia legal se nutre de los conceptos de violencia estructural y simbólica, que se retroalimentan mutuamente; la violencia es a la vez estructural puesto que carece de autores identificables y es simbólica porque su propio carácter de imposición se normaliza. Asimismo, entienden que las diversas formas de violencia estructural y simbólica que están codificadas en las leyes no sólo producen sufrimiento social inmediato, sino que pueden producir perjuicios a largo plazo en aspectos clave del proceso de incorporación de los migrantes. En cuanto al uso del concepto de violencia estructural, las autoras se apoyan y retoman a varios autores, incluyendo al propio Galtung.

La lente de la violencia legal, con su capacidad de capturar la violencia física, estructural y simbólica, tal como posibilita la ley, expone la naturaleza entrelazada de estas formas de violencia, ya que una forma engendra otra, y permite el reconocimiento de las consecuencias violentas de la ley cuando hacen presencia, particularmente cuando éstas se perpetúan e incrustan en estructuras de dominación.

Mientras que Nevins (2005), denunciaba que eran las leyes el mecanismo/instrumento a través del cual los actores estatales generan el infortunio delos migrantes, Menjívary Abrego (2012) dan un paso más 
allá al considerar que la ley amerita una conceptualización particular: violencia legal. Dada la trascendencia que las leyes inmigratorias y referidas a los migrantes tienen sobre la vida de las familias migrantes y sus miembros, la generación de una conceptualización de este tipo permite dar cuenta de las vicisitudes que sufren a causa de las leyes y que quedan invisibilizadas por el carácter estructural y legal de las mismas.

El esquema de violencia legal en Menjívar y Abrego (2012) tiene el mismo problema de amplitud que la propuesta de Galtung, por más que la violencia se delimite a la ley. Por tanto, cabe preguntarse en qué casos debería ser aplicado tal concepto. Las propias autoras utilizan el concepto para analizar como las leyes afectan a aspectos muy diversos de la vida de los migrantes que pueden dificultar las trayectorias de incorporación, extendiendo de manera notable el uso del término violencia. Se podría interpretar que la incorporación exitosa es el estándar que permite observar si se logran las realizaciones posibles, dado que la violencia legal supondría diversas trabas para que esta incorporación se produzca.

Aunque considero acertado no enfatizar el análisis de la violencia estructural en quiénes son los autores de la violencia, ya que como señala Galtung estos pueden incluso no ser identificables, no creo que se deba señalar taxativamente que el caso de la violencia legal no pueda identificarse a los autores tal violencia, en tanto se podría debatir, si por estos se podría entender como los autores y promotores de las leyes, ya sea en grado de autores intelectuales. Al igual que Galtung, considero que no es tan relevante evidenciar a los perpetradores puesto que siempre podemos llegar a la conclusión de que quienes generan y reproducen materialmente las estructuras más concretas donde podemos detectar a autores concretos, lo hacen en función de estructuras represivas más amplias en las cuales "todos seríamos responsables" como sociedad. Resulta más importante dar cuenta de la manera en que se generan y reproducen las estructuras represivas para cambiarlas y con ello evitar sus nefastas consecuencias. Esto no es óbice para que, en determinados casos, se pueda evidenciar cuál es la manera en que ciertas estructuras se crean y mantienen.

Por otra parte, la hiperjudicialización de la vida social y los altos estándares de vida en Estados Unidos hacen apropiado analizar la violencia legal a la manera que lo hacen las autoras. Las leyes generan la condición de ilegalidad/irregularidad de migrantes extranjeros 
(Balibar, 2000; De Genova, 2002 Nevins, 2003, 2005; Mezzadra, 2012) $\mathrm{y}$, en segundo lugar, les someten a regímenes de deportabilidad (De Genova, 2002) y de attrition trough enforcement (Torre Cantalapiedra, 2018), que pueden ser observados bajo la lente de la violencia estructural, y más específicamente de la violencia legal.

Los cientos de migrantes que cada día logran llegar a Estados Unidos de manera indocumentada, incluidos participantes en caravanas de migrantes, no dejan atrás la violencia. No sólo porque ahora enfrentan la violencia legal — posiblemente, más soportable-, sino porque desde una perspectiva familiar, muchos de ellos dejan atrás a parte de sus familiares, padres y hermanos, incluso a hijos y esposas. Familiares que todavía viven el horror de la situación de violencia en Centroamérica y del tránsito por México.

A modo de conclusión: cinco reflexiones sobre violencia estructural y migración.

1) Dados los crecientes nexos entre migración y violencia, se deberá seguir desarrollando la acepción estructural a fin de dar cuenta de las situaciones de violencia que sufren los migrantes en diferentes momentos de sus trayectorias migratorias por diversas geografías mundiales. La noción de violencia estructural posee una gran capacidad para adaptarse a los diferentes contextos sociales y en cada uno de ellos trae al análisis la violencia que sufren los migrantes, estos elementos pasarían desapercibidos en cualquier aproximación que utilizase exclusivamente el concepto de violencia directa. Los trabajos sobre violencia estructural han evidenciado que las estructuras/mecanismos represivos que generan/ causan violencia estructural son de naturaleza muy distinta. Al mismo tiempo consideran, desde estructuras sumamente abstractas, las cuáles aluden a la/s sociedad/es como autor/ es (véase, por ejemplo, la organización del mundo en estadosnación), a estructuras más concretas que permiten señalar a actores específicos que directa o indirectamente ayudan a edificarlas (véanse, las instituciones que a través de sus agentes implementan las leyes). Además, han entendido y explorado de distintas maneras la relación entre violencia estructural y violencia directa, esta última es mejor entendida en relación con la primera. En suma, lo señalado en este párrafo implica 
que el concepto de violencia estructural permite dar una mayor profundidad a los análisis sobre violencia.

2) La ley aparece de manera sobresaliente en los trabajos analizados. Tanto en el derecho internacional como en las legislaciones nacionales, la regulación de la movilidad de las personas resulta de un mecanismo/estructura esencial de producción y reproducción de violencia estructural. Nos resulta natural ver el mundo dividido en estados-nación que poseen la soberanía absoluta sobre sus territorios, por tanto, para decidir a quién dejar entrar y a quién no. Esta organización del mundo puede considerarse un régimen internacional de apartheid global, que impide la movilidad de los más desfavorecidos, y, asimismo, dificulta que operen a nivel global mecanismos más efectivos de redistribución económica y justicia social.

El régimen internacional de refugiados es una construcción legal obsoleta y excluyente que no sólo es ciega a muchas formas de violencia directa, sino que está muy lejos de llegar a plantearse la inclusión de los casos de violencia estructural, por nefastas que sean sus consecuencias en términos de vidas y sufrimiento humano.

Asimismo, la ley genera muchos problemas a los migrantes en los lugares de destino que pueden ser observados desde el punto de vista de la violencia estructural.

3) Si bien los trabajos sobre violencia estructural han abarcado una amplia gama de procesos migratorios, todavía quedan aspectos por escrutar. Entre ellos cabe mencionar dos procesos relacionados con la ley y el poder coercitivo del Estado y su uso "legítimo" de la violencia: la criminalización y la deportación. Además, los desarrollos teóricos obtenidos en el estudio de un determinado proceso migratorio son de utilidad tanto para el estudio de otros aspectos de la migración como para los estudios sobre violencia en otras materias.

4) ¿Son trabajos académicos o discursos políticos pro-migrantes? Como se puede apreciar en todos los trabajos, en mayor o menor grado, retomar el esquema de violencia Galtung sirve para denunciar situaciones en los que la violencia directa no alcanza para referir a situaciones de privación de necesidades básicas de los migrantes y consecuencias muy adversas sobre sus vidas, incluyendo la muerte. Por lo que este tipo de trabajos académicos tiene una faceta política evidente, que siempre 
posee cualquier publicación, pero que aquí es más acuciante al abordar y evidenciar problemas sociales. Es por ello por lo que este tipo de investigaciones pueden llegar a ser tachadas de acientíficas y de ser discursos puramente políticos. Una de las claves para discernir que estamos ante un trabajo académico, y no frente a un panfleto político, es el rigor teórico y metodológico de la investigación.

5) Para lidiar con el problema anunciado en el apartado anterior, se puede afirmar que futuras investigaciones que apliquen el concepto de violencia estructural se pueden ver beneficiadas de seguir los postulados del paradigma de investigación teoría crítica. ${ }^{14}$ Adoptar esta perspectiva de investigación en estos trabajos tiene al menos dos ventajas: I) encaja con las metas de estos - exponer determinados problemas sociales que antes no habían sido vistos como tales-y II) permite manejar adecuadamente los valores del investigador.

\section{REFERENCIAS BIBLIOGRÁFICAS}

Abrego, L. J. y Menjívar C. (2011). "Immigrant Latina Mothers as Targets of Legal Violence", en International Journal of Sociology of the Family, 37(1), pp. 9-26.

Alonso Meneses, G. (2005). "Violencias asociadas al cruce indocumentado de la frontera México-Estados Unidos", en Nueva Antropología, XX (65), pp. 113-129.

Arango, J. (2016). "A través del mediterráneo: tragedia de los refugiados y crisis de la Unión Europea, en Arango, J., Mahía, R., Moya, D. y Sánchez-Montijano, E. (Coords.), El año de los refugiados. Anuario CIDOB de la Inmigración, pp. 30-55, Cidob, Barcelona. Consultado el 12 de enero de 2019 en https://www.cidob.org/es/publicaciones/serie_de_publicacion/anuario_cidob_de_la_inmigracion/el_ano_de_los_refugiados_anuario_cidob_de_la_inmigracion_2015_2016_nueva_epoca

Balibar, E. (2000). What We Owe to the San-Papiers. Consultado el 12 de enero de 2019 en http://eipcp.net/transversal/0313/balibar/en

Calva Sánchez, L. E., Castañeda, A., Coubès, M.-L. y París Pombo, M. D. (2015). Principales Resultados de la Encuesta Sobre Agresión y Abuso a Migrantes (EAAM) devueltos por las autoridades migratorias, 2012, El Colegio de la Frontera Norte, México. Consultado el 20 de enero de 2019 en http://www.colef.mx/wp-content/uploads/2015/03/EAAM_19_marzo. pdf

14 Para más información sobre este paradigma, véase, y Lincoln (1994). 
Castañeda, J. G. (2019). “El retroceso exterior de México", ElPaís.com. Consultado el 29 de enero de 2019 en https://elpais.com/elpais/2019/01/25/ opinion/1548441758_092877.html

Castles, S. y Miller, M. J. (2004). La era de la migración: movimientos internacionales de población en el mundo moderno, Universidad Autónoma de Zacatecas / Secretaria de Gobernación / Instituto Nacional de Migración / Fundación Colosio / Miguel Ángel Porrúa, México. Consultado el 12 de enero de 2019 en http://biblioteca.diputados.gob.mx/janium/bv/ce/ scpd/LIX/era_mig.pdf

Crisp, J. (2003). A new asylum paradigm? Globalization, migration and the uncertain future of the international refugee regime. Consultado el 15 de diciembre de 2018 en https://www.unhcr.org/research/working/3fe16d835/new-asylum-paradigm-globalization-migration-uncertain-future-international.html

Cordero Díaz, B. L. y Garibo García, M. G. (2018). “Violencias encarnadas: migración de mujeres centroamericanas", pp. 171-194, en López Castellanos, N. (coord.), Procesos migratorios en la Centroamérica del siglo XXI, UNAM / La Biblioteca, México.

Cortés, A. (2018). "Violencia de género y frontera: migrantes centroamericanas en México hacia los EEUU", en Revista Europea de Estudios Latinoamericanos y del Caribe, No. 105, pp. 39-60.

Cuellar Díaz, N. A. y Moreno Hernández, H. C. (2018). "Atención a la población en situación de movilidad en el espacio transfronterizo México-Guatemala, pp. 123-146, en López Castellanos, N. (coord.), Procesos migratorios en la Centroamérica del siglo XXI, UNAM / La Biblioteca, México.

De Genova, N. P. (2002). "Migrant "Illegality" and Deportability in Everyday Life", en Annual review of anthropology, 31(1), pp. 419-447.

Delgado Wise, R., Márquez Covarrubias, H. y Puentes, R. (2013). "Reframing the Debate on Migration, Development and Human Rights", en Population, Space and Place, 19(4), pp. 430-443.

El Colef. (2018). La caravana de migrantes centroamericanos en Tijuana 2018. Diagnóstico y propuestas de acción. El Colegio de la Frontera Norte, Tijuana. Consultado el 13 de enero de 2019 en https://www.colef.mx/wp-content/uploads/2018/12/documento-caravana-migrante-3dic18-2.pdf

Espinar Ruiz, E. (2010). "Migrantes y refugiados: reflexiones conceptuales", en Obets. Revista de Ciencias Sociales, 5(1), pp. 35-47.

Farmer, P. (2005). Pathologies of Power: Health, Human Rights, and the New War on the Poor, University of California Press, Berkeley.

Galtung, J. (1995). "Violencia, paz e investigación sobre la paz", en Investigaciones teóricas. Sociedad y cultura contemporáneas, Johan Galtung (au- 
tor), pp. 310-354, Instituto de Cultura <<Juan Gil-Albert $>>$ y Ed. Tecnos, Madrid.

Galtung, J. (2003). "Violencia Cultural", en Documentos de trabajo Gernika Gogorutz, No. 14, pp. 1-28.

Giorguli Saucedo, S. E., García Guerrero, V. y Masferrer, C. (2018). Un sistema migratorio en construcción. Dinámica demográfica y políticas migratorias en Norteamérica y el Triángulo Norte de Centroamérica, Centro de Estudios Demográficos, Urbanos y Ambientales, El Colegio de México, México. Consultado el 14 de enero de 2019 en https://cedua.colmex.mx/ amsitm.html

Guba, E. G. y Lincoln, Y. (1994). "Competing paradigms in qualitative research", en Denzin, N. K. y Lincoln Y. S. Handbook of Qualitative Research (pp. 105-117), Sage Publications, Thousand Oaks, California.

Heyman, J. y Slack J. (2018). Blockading Asylum Seekers at Ports of Entry at the US-Mexico Border Puts Them at Increased Risk of Exploitation, Violence, and Death, Center for Migration Studies. Consultado el 15 de enero de 2019 en http://cmsny.org/publications/heyman-slack-asylum-poe/

INE. (2018). Hogares en condición de pobreza 2016, Instituto Nacional de Estadística de Honduras, Honduras. Consultado el 14 de enero de 2019 en https://www.ine.gob.hn/index.php/25-publicaciones-ine/91-linea-de-pobreza

Izcara Palacios, S. P. (2012). "Violencia contra inmigrantes en Tamaulipas", en European Review of Latin American and Caribbean Studies, No. 93, pp. 3-24.

Izcara Palacios, S. P. (2016). "Violencia postestructural: migrantes centroamericanos y cárteles de la droga en México", en Revista de Estudios Sociales, No. 56, pp. 12-25.

Jácome, F. (2008). Trans-Mexican Migration: a Case of Structural Violen$c e$, London School of Economics. Consultado el 15 de enero de 2019 en http://pdba.georgetown.edu/CLAS\%20RESEARCH/Working\%20Papers/WP2.pdf

La Parra, D. y Tortosa J. M. (2003). “Violencia estructural: una ilustración del concepto", en Documentación Social, No. 131, pp. 57-72.

Martínez, D. E., Reineke, R. C., Rubio-Goldsmith, R y Parks, B. O. (2014). "Structural violence and migrant deaths in Southern Arizona: Data from the Pima County office of the medical examiner, 1990-2013", en Journal on Migration and Human Security, 2(4), pp. 257-286.

Menjívar, C. y Abrego, L. J. (2012). "Legal Violence: Immigration Law and Lives of Central American Immigrants", en American Journal of Sociology, 117(5), pp. 1380-1421.

Mezzadra, S. (2012). "Capitalismo, migraciones y luchas sociales: La mirada de la autonomía", en Nueva Sociedad, No. 237, pp. 159-179. 
MSF. (2017). Forzados a huir del Triángulo Norte de Centroamérica: una crisis humanitaria olvidada, Médicos Sin Fronteras. Consultado el 20 de enero de 2019 en https://www.msf.mx/document/forzados-a-huir-deltriangulo-norte-de-centroamerica-una-crisis-humanitaria-olvidada

Nájera Aguirre, J. N. (2016). "El complejo estudio de la actual migración en tránsito por México: Actores, temáticas y circunstancias", en Migraciones Internacionales, 8(3), pp. 255-266.

Nevins, J. (2003). "Thinking Out of Bounds: A Critical Analysis of Academic and Human Rights Writings on Migrant Deaths in the US-Mexico Border Region", en Migraciones Internacionales, 2(2), pp. 171-190.

Nevins, J. (2005). "A Beating Worse than Death: Imagining and Contesting Violence in the U.S.-Mexico Borderlands", en AmeriQuests, 2(1), pp. 1-25. Consultado el 10 de noviembre de 2017 en http://ejournals.library.vanderbilt.edu/ojs/index.php/ameriquests/article/view/64

París Pombo, M. D. (2017). Violencias y migraciones centroamericanas en México, El Colegio de la Frontera Norte, México.

Pogge, T. (2010). "Migraciones y Pobreza”, en Arbor Ciencia, Pensamiento y Cultura, No. 744, pp. 571-583.

REDODEM. (2017). El Estado Indolente. Violencia en las rutas migratorias, México, REDODEM.

Reineke, R. y Martínez, D. E. (2014.). "Migrant Deaths in the Americas (United States and Mexico)", en Brian, T. y Laczko, F. (eds.), Fatal Journeys. Tracking Lives Lost during Migration, pp. 45-83, OIM, Ginebra. Consultado el 10 de marzo de 2019, en https://publications.iom.int/es/books/fataljourneys-tracking-lives-lost-during-migration

Rocha Romero, D. y Vera Martínez, M. C. (2016). "Exconvictos deportados, violencia y cooperación transfronteriza entre México y Estados Unidos", en CONfines, año 12(23), pp. 63-83.

Silva A. C. y Massey, D. S. (2015). "Violence, Networks, and International Migration from Colombia", en International Migration, 53(5), pp. 162-178.

Silva Hernández, A. (2015). "Estrategias de tránsito de adolescentes centroamericanos independientes: enfrentando la frontera vertical en México", en REMHU, Revista Interdisciplinar da Mobilidade Humana, No. 44, pp. 99-117.

Slack, J. y Whiteford, S. (2010). "Viajes violentos: la transformación de la migración clandestina hacia Sonora y Arizona”, en Norteamérica, 5(2), pp. 79-107.

Spener, D. (2008). "El apartheid global, el coyotaje y el discurso de la migración clandestina: distinciones entre violencia personal, estructural y cultural", en Migración y Desarrollo, No. 10, pp. 127-156. 
TRAC. (2018). Asylum Decisions and Denials Jump in 2018. Consultado el 18 de enero de 2019) en https://trac.syr.edu/immigration/reports/539

Torre Cantalapiedra, E. (2018). "La estrategia de attrition through enforcement: políticas antiinmigrantes de Arizona y sus efectos sobre las familias mexicanas", en Norteamérica, Revista Académica del CISAN-UNAM, año. 13, No. 1, pp. 169-192.

Torre Cantalapiedra, E y Giorguli, S. E. (2016). "Movilidades internas e internacionales en Colombia: determinantes, patrones migratorios y diversidad de destinos, 1950-2010", en Revista Latinoamericana de Estudios de Población (RELAP), año 10, No. 19, pp. 7-32. Consultado el 11 de diciembre de 2018 en http://revistarelap.org/ojs/index.php/relap/article/view/151

Torre Cantalapiedra, E. y Yee Quintero, J. C. (2018). “México ¿una frontera vertical? Políticas de control del tránsito migratorio irregular y sus resultados", en LiminaR. Estudios Sociales y Humanísticos, vol. XVI, No. 2, pp. 87-104.

Torre Cantalapiedra, E. y Mariscal Nava, D. M. (s.f.). "Batallando con fronteras: estrategias migratorias en tránsito de participantes en las caravanas de migrantes".

Varela Huerta, A. (2017). "La trinidad perversa de la que huyen las fugitivas centroamericanas: violencia feminicida, violencia de estado y violencia de mercado", en Debate Feminista, No. 53, pp. 1-17.

Vogt, W. (2013). "Crossing Mexico: Structural Violence and the Commodification of Undocumented Central American Migrants", en American Ethnologist, 40(4), pp. 764-780.

Willers, S. (2016). "Migración y violencia: las experiencias de mujeres migrantes centroamericanas en tránsito por México", en Sociológica, 31(89), pp. 163-195.

Yee Quintero, J. C. (2016). Las víctimas del estado: violencia hacia migrantes hondureños, perpetrada por agentes del estado, durante su tránsito por México. Tijuana: El Colegio de la Frontera Norte, tesis de maestría. Consultado el 11 de diciembre de 2019 en https://www.colef.mx/posgrado/ wp-content/uploads/2016/11/TESIS-Yee-Quintero-Jos\%C3\%A9-Carlos. pdf

Yee Quintero, J. C. y Torre Cantalapiedra, E. (2016). "Lidiando con la frontera vertical: estrategias de hondureños en tránsito por México", REMHU: Revista interdisciplinar da mobilidade huamana, año XXIV, No. 46, pp. 97114.

Zolberg, A. R., Suhrke, A. y Aguayo, S. (1989). Escape from violence. Conflict and the Refugee Crisis in the Developing Countries, Oxford University Press, Oxford. 


\section{RESUMEN CURRICULAR DEL AUTOR}

Eduardo Torre Cantalapiedra

Doctor en Estudios de Población por El Colegio de México. Entre sus publicaciones recientes se encuentran los artículos "Explaining State and Local Anti-Immigrant Policies in the United States: The Case of Arizona's SB 1070" en Migraciones Internacionales, vol. 8, 2016 y "Viviendo en las sombras: estrategias de adaptación de familias inmigrantes mexicanas en Arizona, 2007-2015" en Papeles de Población, vol. 22, 2016, en coautoría con María Eugenia Anguiano Téllez. Actualmente, es investigador posdoctoral en el Instituto de Investigaciones Jurídicas de la Universidad Nacional Autónoma de México.

Dirección electrónica: e.torreca@gmail.com 